\title{
Self-Oscillating, Third-Harmonic Mode-Locked 1-GHz Nd:YLF Laser
}

\author{
Tae Jun Yu, Jai Hee Sung, and Chang Hee Nam \\ Department of Physics and Center for Electro-Optics, Korea Advanced Institute of Science and Technology, \\ Taejon 305-701, KOREA
}

\begin{abstract}
A Nd:YLF laser has been actively mode-locked at the third-order harmonic cavity frequency using a lithium niobate phase modulator. Self-oscillating pulses of 14 ps duration, $1 \mathrm{GHz}$ repetition rate, and $10 \mathrm{~mW}$ average power at $1053 \mathrm{~nm}$ wavelength have been obtained. A $1 \mathrm{GHz} \mathrm{rf}$ signal to drive a phase modulator was obtained by using $1 \mathrm{GHz}$ pulse train without $\mathrm{rf}$ synthesizer as a driver of an active mode locker.
\end{abstract}

\section{INTRODUCTION}

AM and FM active mode-locking methods have been utilized to generate high-quality pulse trains at high repetition rate in several laser systems, including semiconductor lasers, and erbium-doped fiber lasers. However, these techniques usually fail to produce pulse trains with long-term stability $[1,2]$. Some forms of active stabilization are generally required for modelocked lasers to maintain exact synchronism between the modulation frequency and the natural cavity repetition rate, which tends to drift in time owing to mechanical vibrations and thermal changes. Additionally, because the higher repetition rate makes the cavity length short, it is difficult to install the mechanical mountings of an active mode-locker close to an output mirror for the maximum modulation efficiency in a bulk laser system. For these problems in high repetition rate, active mode-locking lasers, a direct drive method [3] and a harmonic mode-locking method [4] may be useful. The direct drive method, which would automatically track any change in cavity length and provide a correct modulation frequency, can solve the synchronization problem and does not require an rf synthesizer as a driver of the phase modulator. A harmonic mode-locked laser can relax the short cavity requirement for a high repetition rate laser.

\section{EXPERIMENTAL SETUP}

We developed a self-oscillating, third-harmonic mode-locked Nd:YLF laser operating at a $1 \mathrm{GHz}$ repetition rate by using the direct drive, harmonic modelocking method. The experimental arrangement of the laser is shown in Fig. 1. The Nd:YLF of active medium of a $10 \mathrm{~mm}$ long rod with a $4 \mathrm{~mm}$ radius was longitudinally pumped by a LD $3 \mathrm{~W}$ GaAlAs diode (SDL-2482P1). The output was first collimated with a lens of 6.5 $\mathrm{mm}$ focal length (Melles Griot 06GLC001/D), passed

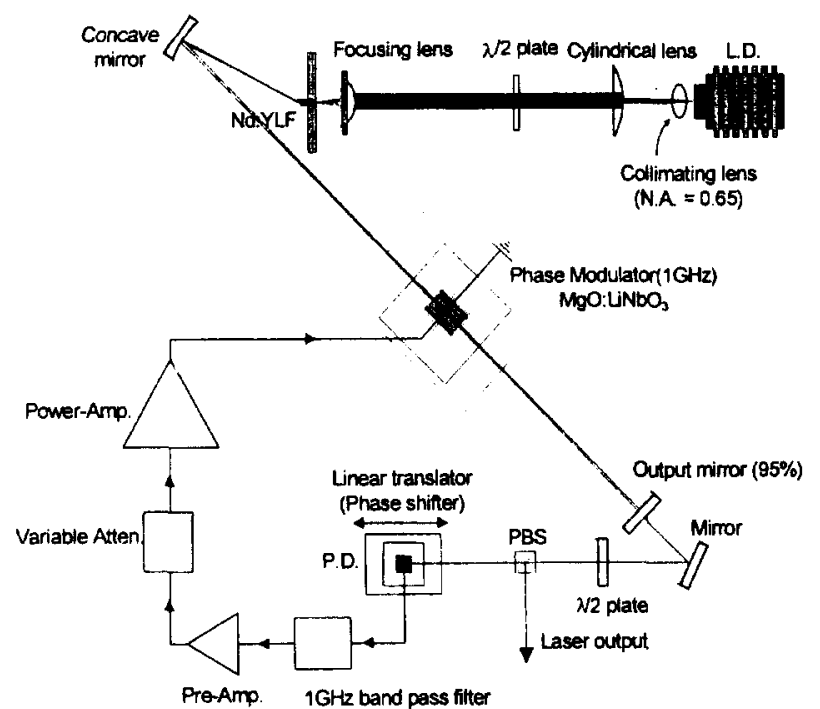

FIG. 1. Schematic layout of the third harmonic mode-locked laser system PBS; polarized beam splitter. $\mathrm{PD}$; high speed photodetector (bandwidth of $2 \mathrm{GHz}$ ).

through a plano-cylindrical lens of $150 \mathrm{~mm}$ focal length to provide a nearly parallel beam and through a half wave plate to achieve optimum pumping efficiency, and finally focused by a lens of $25 \mathrm{~mm}$ focal length on the laser rod. One side of the laser rod is dichroic coated for high reflectivity at the laser wavelength of $1053 \mathrm{~nm}$ $(\mathrm{R}>99.8 \%)$ and antireflection coated at the pump wavelength $800 \mathrm{~nm}(\mathrm{~T}>90 \%)$. The second side of the laser rod is a Brewster cut face. The laser cavity includes a folding mirror with a $0.15 \mathrm{~m}$ radius of curvature tilted by 23 degree to the resonator axis and an output mirror with a $5 \mathrm{~m}$ radius of curvature and $5 \%$ transmission at the laser wavelength. To compenste astigmatism, the concave mirror was tilted by considering the following equation.

$$
\frac{d}{n}\left(1-\frac{1}{n^{2}}\right)=f \sin (\theta) \tan (\theta),
$$


where the refractive index is 1.45 , the length of active medium is $8.6 \mathrm{~mm}$ and the focal length of the concave mirror is $75 \mathrm{~mm}$. So the laser cavity astigmatism is compensated. The phase modulator (New Focus Model 4423) consists of a Z-cut, Y-propagating, 20 $\mathrm{mm}$ long $\mathrm{MgO}: \mathrm{LiNbO}_{3}$ which has high maximum optical power limit $\left(4 \mathrm{~W} / \mathrm{mm}^{2}\right)$ and low optical-insertion loss. The laser polarization by a Brewster cut face of the laser rod and an electric field for modulation was paralleled with the optical Z-axis of electro-optic crystal. The electric field was produced in a high $Q$ resonant cavity with a resonance frequency of $1 \mathrm{GHz}$. The effective cavity length was set to $45 \mathrm{~cm}$, which is three times longer than the fundamental cavity length corresponding to the $1 \mathrm{GHz}$ modulation frequency.

For a best modulation efficiency, the phase modulator needs to be properly located in the laser cavity. The position of the electro-optic crystal may be decided by considering the following equations [5]

$$
\begin{gathered}
\delta_{m}=\frac{2 \pi}{\lambda}\left(\frac{n_{e}^{3} r_{33}}{2}\right) \frac{l}{d} V, \\
\delta=\frac{L}{N l} \frac{2}{\pi}\left(\sin \frac{N l}{L} \frac{\pi}{2}\right)\left(\cos \frac{N z_{0} \pi}{L}\right) \delta_{m},
\end{gathered}
$$

where $\lambda$ is the wavelength of the laser, $\delta_{m}$ is the optical phase shift obtained by applying a voltage $V$ across the electro-optic crystal with a length $l$, a width $d$ of the electro-optic phase modulator, $\delta$ is the optical phase shift in the laser cavity, $N$ is the harmonic order or the number of mode-locked pulses in the laser cavity, and $z_{0}$ is the position of the center of the crystal from the output mirror. Here the length of laser cavity $L$ is 45 $\mathrm{cm}$, the length of the electro-optic crystal $l$ is $2 \mathrm{~cm}$, and $N$ is 3 in the third harmonic mode-locking laser system, so the optical phase shift in the laser cavity is approximately $\delta \cong \cos \left(N \pi z_{0} / L\right) \cdot \delta_{m}$ from Eq.(2). To achieve the maximum modulation efficiency, the modulator has to be located at a distance $z_{0}(0, L / 3,2 L / 3, L)$ from the output mirror. Because the mechanical mountings of the phase modulator did not allow putting the modulator close to the output mirror, we chose a distance $d$ $=15 \mathrm{~cm}$.

\section{DIRECT DRIVE AND HARMONIC MODE-LOCKING METHOD}

To achieve a third harmonic mode-locking system without an rf synthesizer which is a driver of an active mode-locker, we choose the third beat frequency $3 c / 2 L$ of the laser oscillator as an $\mathrm{rf}$ drive signal by using a high-speed photodetector (EOT-2000) with a bandwidth of $2 \mathrm{GHz}$. Fig. 2. is the r.f. power spectrum of c.w. fluctuation captured by fast photodetec-

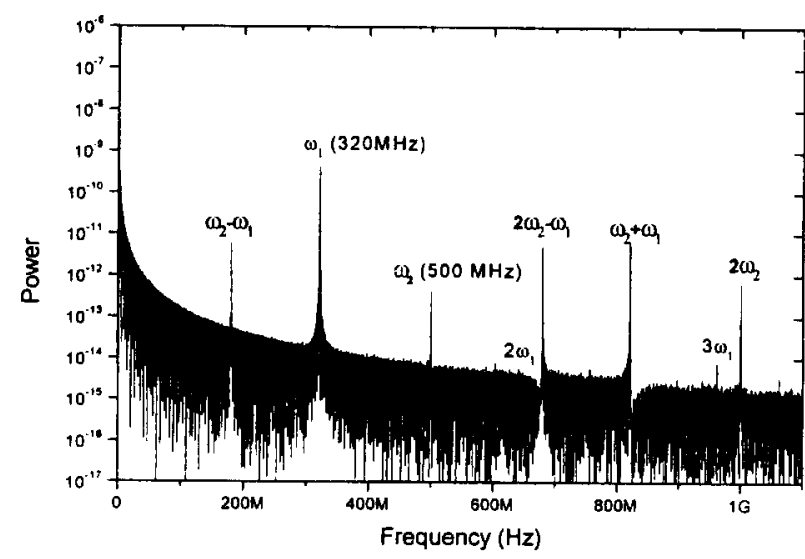

FIG. 2. R.f. power spectrum of the c.w. fluctuation $\left(\omega_{2}, 2 \omega_{2}, \omega_{2}-\omega_{1}, 2 \omega_{2}-\omega_{1}, \omega_{2}+\omega_{1}\right.$ are dummy peaks by aliasing of a digital oscilloscope).

tor with a $500 \mathrm{MHz}$ digital oscilloscope. There exists the third harmonic beat $(3 c / 2 L)$ in this figure. Other peaks are dummy frequency by aliasing with the 500 $\mathrm{MHz} \operatorname{clock}\left(\omega_{2}\right)$ of the digital oscilloscope, but the natural cavity frequency $\left(\omega_{1}\right)$ and the harmonic frequency are true. We believe that the interaction of amplified spontaneous emission and Fabry-Perot laser cavity generates the third beat frequency $3 c / 2 L$. This signal was amplified by the rf amplifier(Minicircuits ZHL1000-3W) with $38 \mathrm{~dB}$ gain, properly phase-shifted by an optical delay line, and then applied to the phase modulator. The adjustable phase shifter compensates for the delay in the feedback loop. When the phase of the electric signal on the phase modulator and the signal derived from the laser equal an integer times $\pi$, the feedback loop goes into regeneration and the laser goes to mode-locked operation [3]. Therefore the interval between one phase matching position and another of the phase shifter was $15 \mathrm{~cm}$ owing to ambiguity in the phase relationship between the $1 \mathrm{GHz}$ laser pulse train and the modulator driving signal, as the pulses may pass through the modulator at either of the two extreme voltage points. The resultant mode-locking system was a closed-loop oscillator using the third beat frequency of the laser cavity. Such a system would automatically track any change in cavity length. In the general method, the laser behavior changes from FM operation to FM mode-locking by tuning an rf signal frequency of a synthesizer. In the direct drive method, however, the laser behavior directly goes to FM modelocking.

\section{RESULTS AND DISCUSSION}

The output of the laser was monitored in the time domain by a fast photodiode (New Focus Model 1434) with a rise time of $17 \mathrm{ps}$ connected to a sampling os- 

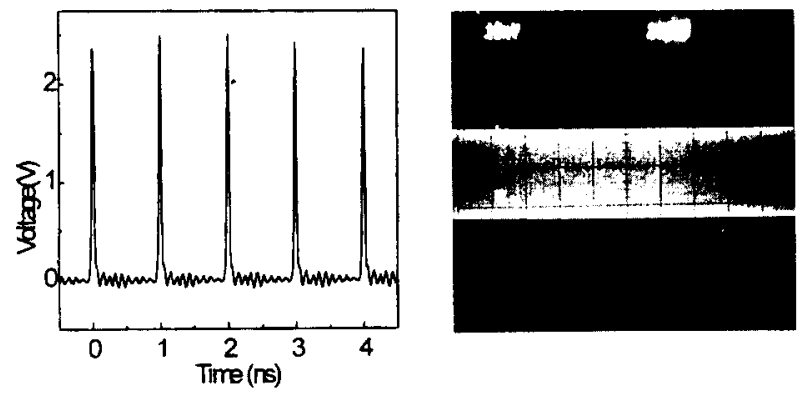

FIG. 3. Temporal monitoring of the $1 \mathrm{GHz}$ Nd:YLF laser output using (a) a sampling scope (span : 5 ns) and (b) an analog oscilloscope (span : $2 \mathrm{~s}$ ).

cilloscope (HP 58121A) with a bandwidth of $20 \mathrm{GHz}$. The resulting pulse train is shown in Fig. 3(a). A stable mode-locked pulse train was monitored during thirty minutes with an analog oscilloscope with a bandwidth of $1 \mathrm{GHz}$. The resulting pulse train is shown in Fig. 3(b). As the phase of the rf signal and the laser pulse was synchronized on the phase modulator, temporal structure of the laser output is shown in Fig. 4(a) which has three pulses in the laser cavity. However, as the phase of the rf signal and the laser pulse was not synchronized on the phase modulator, tem-

(a)

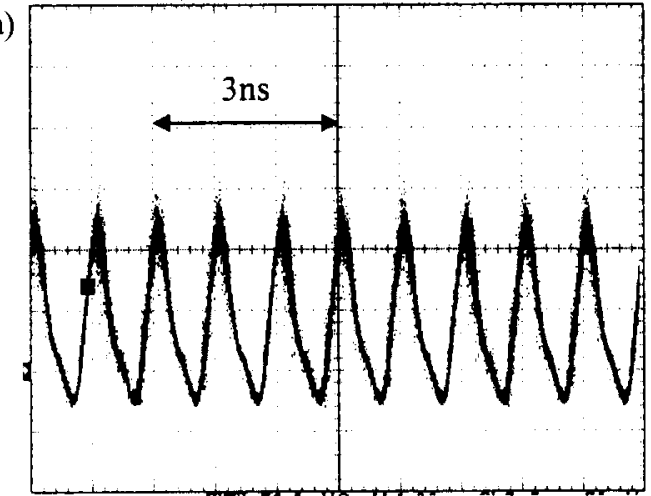

(b)

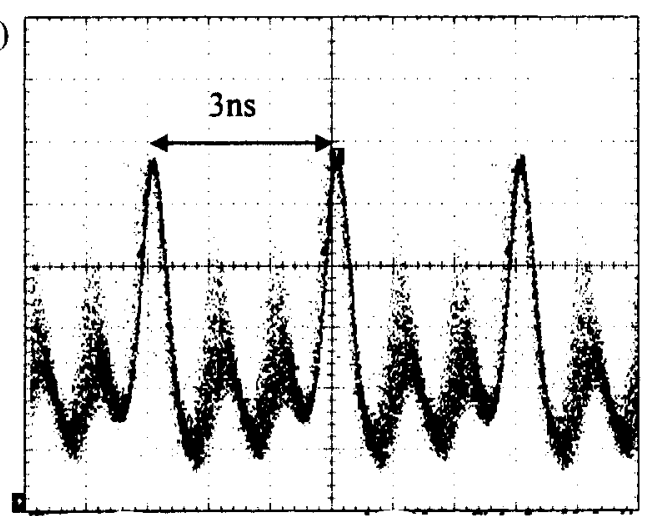

FIG. 4. Temporal monitoring of the $1 \mathrm{GHz}$ Nd:YLF laser oscillator with (a) a well phase matched case, (b) an slightly mismatched case. Horizontal scale, 1ns/divisiontely phase matched case. poral structure of the laser output has been changed [see Fig. 4(b)] with intent to have one pulse in the laser cavity. With the pumping configuration and in $\mathrm{CW}$ operation, the maximum output power of $200 \mathrm{~mW}$ at the pump power level of $1.4 \mathrm{~W}$ was obtained, with a threshold power of $105 \mathrm{~mW}$. The maximum output power was limited by the damage thresholds of the $\mathrm{Mg}: \mathrm{LiNbO}_{3}$ phase modulator. When we increased the pump power to the laser rod, the stability of the laser system was degraded. So, the maximum output laser power of a stable mode-locked pulse train is $10 \mathrm{~mW}$ with the pump power of $170 \mathrm{~mW}$ because all modes of the mode-locked laser would drift with respect to the center of the atomic line owing to thermal changes of the laser rod from pump power. The phase and the amplitude of the first beat with respect to the phase modulator (the third beat with respect to the laser cavity) change abruptly as the center of all modes of the mode-locked laser move from one side of an atomic line to the other [6]. Because of this problem, we would utilize the second-order beat frequency in the future instead of the first beat with respect to the phase modulator, to avoid the failure mode which occurs due to a spontaneous switch of $180^{\circ}$ in the phase of the output pulse train $[5,6]$. The rf power spectrum of the laser was shown in Fig. 5(a). The power ratio

(a)

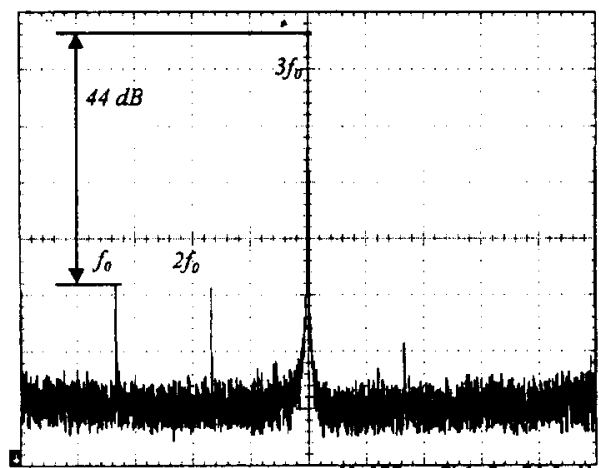

(b)

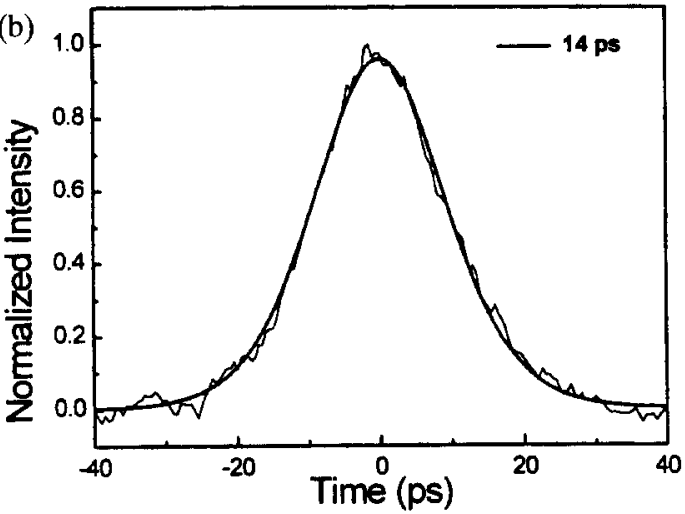

FIG. 5. (a) The rf power spectrum (peak frequency : $1.00 \mathrm{GHz}$ ) from the third harmonic mode-locked Nd:YLF laser oscillator. $f_{0} ; c / 2 L$. (b) Measured collinear auto-correlation trace. 
between the third beat and the first beat with respect to the laser cavity is $44 \mathrm{~dB}$. As the position of the phase shifter was optimized, the first beat power was minimized. The pulse duration was measured by using a collinear autocorrelation technique. The measured auto-correlation trace is shown in Fig. 5(b). The pulse duration, for a $\operatorname{sech}^{2}$ pulse shape, was 14 ps.

\section{CONCLUSIONS}

In conclusion, we have firstly demonstrated picosecond pulse generation at a high repetition rate of a diode-pumped Nd:YLF laser in a third harmonic cavity without rf synthesizer, using an electro-optic phase modulator $\mathrm{MgO}: \mathrm{LiNbO}_{3}$. Pulses with an average power of $10 \mathrm{~mW}$ and a pulse duration of $14 \mathrm{ps}$ at the wavelength of $1053 \mathrm{~nm}$ were obtained. The combination of harmonic mode-locked method and direct drive method has advantages for solid state laser systems requiring high repetition rate and long term stability in communication.

\section{ACKNOWLEDGMENTS}

This research was supported by Center for ElectroOptics (CEO) and Agency for Denfence Development (ADD)

\section{REFERENCES}

[1] T. Harvey and L.F. Mollenauer, Opt. Lett. 18, 107 (1993).

[2] M. Nakazawa, E. Yoshida, and Y. Kimura, Electron. Lett. 30, 1603 (1994).

[3] W. Koechner, Solid-State Laser Engineering, (Springer, 4th ed., 1996), p. 540.

[4] S. Longhi et al., Opt. Lett. 19, 1985 (1994).

[5] S. E. Harris and O. P. McDuff, IEEE J. Quantum Electron. QE-1, 245 (1965)

[6] S. E. Harris, Appl. Optics 5, 1639 (1966). 